\title{
The structure of the protoplanetary disk surrounding three young intermediate mass stars ${ }^{\star}$
}

\section{Spatially resolved dust and gas distribution}

\author{
D. Fedele ${ }^{1,2,3}$, M. E. van den Ancker ${ }^{3}$, B. Acke ${ }^{4}$, G. van der Plas ${ }^{3,5}$, R. van Boekel ${ }^{1}$, \\ M. Wittkowski ${ }^{3}$, Th. Henning ${ }^{1}$, J. Bouwman $^{1}$, G. Meeus ${ }^{6}$, and P. Rafanelli ${ }^{2}$ \\ 1 Max Planck Institut für Astronomie, Königstuhl 17, 69117 Heidelberg, Germany \\ 2 Dipartimento di Astronomia, Universitá degli studi di Padova, Vicolo dell'Osservatorio 2, 35122 Padova, Italy \\ 3 European Southern Observatory, Karl Schwarzschild Strasse 2, 85748 Garching bei München, Germany \\ 4 Instituut voor Sterrenkunde, KU Leuven, Celestijnenlaan 200B, 3001 Leuven, Belgium \\ Sterrenkundig Instituut “Anton Pannekoek”, University of Amsterdam, Kruislaan 403, 1098 SJ Amsterdam, The Netherlands
} e-mail: dfedele@mpia.de

${ }^{6}$ Astrophysikalisches Institut Potsdam, 14482 Potsdam, Germany

Received 5 May 2008 / Accepted 3 September 2008

ABSTRACT

\begin{abstract}
Aims. We present the first direct comparison of the distribution of the gas, as traced by the [OI] $6300 \AA$ emission, and the dust, as traced by the $10 \mu \mathrm{m}$ emission, in the planet-forming region of proto-planetary disks around three intermediate-mass stars: HD 101412 , HD 135344 B and HD 179218.

Methods. $N$-band visibilities were obtained with VLTI/MIDI. Simple geometrical models are used to compare the dust emission to high-resolution optical spectra in the $6300 \AA$ [O I] line of the same targets.

Results. HD 101412 and HD 135344 B show compact $(<2$ AU) $10 \mu \mathrm{m}$ emission while the [OI] brightness profile shows a double peaked structure. The inner peak is strongest and is consistent with the location of the dust, the outer peak is fainter and is located at 5-10 AU. In both systems, spatially extended PAH emission is found. HD 179218 shows a double ring-like $10 \mu \mathrm{m}$ emission with the first ring peaking at $\sim 1 \mathrm{AU}$ and the second at $\sim 20 \mathrm{AU}$. The [O I] emitting region is more compact, peaking between 3-6 AU. Conclusions. The disks around HD 101412 and HD 135344 B appear strongly flared in the gas, but self-shadowed in the dust beyond $\sim 2$ AU. The difference in the gas and dust vertical structure beyond 2 AU might be the first observational evidence of gas-dust decoupling in protoplanetary disks. The disk around HD 179218 is flared in the dust. The $10 \mu \mathrm{m}$ emission emerges from the inner rim and from the flared surface of the disk at larger radii. No dust emission is detected between $\sim 3-15$ AU. The oxygen emission seems also to come from a flared structure, however, the bulk of this emission is produced between $\sim 1-10$ AU. This could indicate a lack of gas in the outer disk or could be due to chemical effects which reduce the abundance of $\mathrm{OH}$ - the parent molecule of the observed [O I] emission - further away from the star. It may also be a contrast effect if the [O I] emission is much stronger in the inner disk. We suggest that the three systems, HD 179218, HD 135344 B and HD 101412, may form an evolutionary sequence: the disk initially flared becomes flat under the combined action of gas-dust decoupling, grain growth and dust settling.
\end{abstract}

Key words. stars: pre-main sequence - stars: circumstellar matter - stars: planetary systems: protoplanetary disks

\section{Introduction}

Many pre-main-sequence stars are characterized by excess infrared emission above the stellar photospheric level which, depending on the evolutionary state of the system, may start in the near infrared $(1-5 \mu \mathrm{m})$ or at longer wavelengths. The dust distributed around the young star is responsible for this emission. The dust particles absorb a large fraction of the short wavelength stellar photons and re-emit them at infrared wavelengths. This dust is believed to be confined to a disk-like structure which forms in the early phase of star formation as a result of the conservation of the angular momentum of the parental cloud. Disk formation is followed by a longer phase of disk accretion during which disk material accretes onto the young star at a typical accretion rate of $10^{-7}-10^{-10} M_{\odot} \mathrm{yr}^{-1}$. The interstellar dust and gas that forms the disk undergoes changes in its composition and

* Based on observations collected at the European Southern Observatory, Paranal, Chile (Proposal ID: 077.C-0521, 077.C-0263). size. Infrared surveys show that after a mean age of $3 \mathrm{Myr}$ the inner part of the disk is cleared of dust. Viscous accretion, photoevaporation and planet formation are the likely mechanisms responsible for this phenomenon (see review in Henning 2008).

A circumstellar disk is believed to be the locus where planet formation takes place. The large number of recently discovered exo-planetary systems and the variety of such systems raised many new questions about the structure and evolution of protoplanetary disks. Of particular interest for the understanding of disk evolution and planet formation is the coupling of gas and dust in disks. A main assumption underlying essentially all proto-planetary disk models is that dust and gas are thermally coupled. It is an open question whether this assumption holds on the disk surface and efforts have been made to improve disk models by taking into account the dust-gas decoupling (e.g. Kamp \& Dullemond 2004). While gas and dust are thermally coupled in the disk interior, in the low density environments of the disk surface layer, the two components may decouple. 
Table 1. Properties of the programme stars.

\begin{tabular}{|c|c|c|c|c|c|c|c|c|c|c|}
\hline 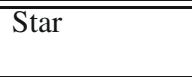 & $\begin{array}{l}\text { RA } \\
(\mathrm{J} 2000)\end{array}$ & $\begin{array}{l}\text { Dec } \\
(\mathrm{J} 2000)\end{array}$ & Sp.T & $\begin{array}{l}M_{\text {star }} \\
{\left[M_{\odot}\right]}\end{array}$ & $\begin{array}{l}F_{12 \mu \mathrm{m}} \\
{[\mathrm{Jy}]}\end{array}$ & $\begin{array}{l}\text { Distance } \\
{[\mathrm{pc}]}\end{array}$ & Meeus group & $\begin{array}{l}\mathrm{PA} \\
{\left[{ }^{\circ}\right]}\end{array}$ & $\begin{array}{l}\text { Inclination } \\
{\left[{ }^{\circ}\right]}\end{array}$ & $\begin{array}{l}{[\mathrm{O} \mathrm{I}] \text { extent }} \\
{[\mathrm{AU}]}\end{array}$ \\
\hline HD 101412 & $11: 39: 44.46$ & $-60: 10: 27.7$ & A0IIIe & 2.3 & 3.22 & 160 & II & - & 30 & $0.15-10$ \\
\hline HD 135344 B & $15: 15: 48.44$ & $-37: 09: 16.0$ & $\mathrm{~F} 4 \mathrm{Ve}$ & 1.7 & 1.59 & 140 & I & 100 & 45 & $0.1-100$ \\
\hline
\end{tabular}

The column "Meeus group" gives the classification in flared (group I) and self-shadowed (group II) disks by Meeus et al. (2001). Column "[O I] extent" reports the extent (minimum and maximum radius) of the [O I] emitting region as derived in Paper I. The disk position angle (PA) and inclination are taken from Paper I.

The detailed structure of the disk surface temperature in the presence of gas-dust decoupling was studied by Jonkheid et al. (2004), Kamp \& Dullemond (2004) and Nomura \& Millar (2005). Different heating/cooling processes act at different heights in the disk. In particular, very high in the atmosphere, with particle densities as low as $n<10^{5} \mathrm{~cm}^{-3}\left(A_{V} \lesssim 10^{-3} \mathrm{mag}\right)$, the gas temperature is set by the balance of photoelectric heating and fine structure line cooling of neutral oxygen (Jonkheid et al. 2004; Kamp \& Dullemond 2004). In the upper layers of protoplanetary disks the gas temperature may exceed the dust temperature. At small radii $(<50 \mathrm{AU})$ the temperature of the gas above the disk photosphere may reach $\sim 10^{4} \mathrm{~K}$. At larger radii ( $>50 \mathrm{AU}$ ) the gas can become as hot as a few hundred Kelvin (Kamp \& Dullemond 2004). However, it is not obvious that such a hot and tenuous disk atmosphere can remain as a stable structure of a disk. The difference in gas and dust temperature as well as other processes (e.g. disk wind, disk evaporation, dust coagulation and settling) may lead to a physical decoupling of gas and dust in disks.

In this paper we present the first direct comparison of the dust and gas emission of three pre-main-sequence stars: HD 101412, HD 135344 B and HD 179218. These are intermediate-mass $\left(1.7-2.7 M_{\odot}\right)$ stars belonging to both group I (flaring disk: HD $135344 \mathrm{~B}$ and HD 179218) and group II (flattened disk: HD 101412) according to the classification of Meeus et al. (2001). In a previous paper (van der Plas et al. 2008, hereafter Paper I) we presented high resolution spectroscopy $\left(\frac{\lambda}{\Delta \lambda}=\right.$ 77 000) of the optical [O I] $6300.304 \AA$ line with VLT/UVES of these three stars. Here we present $N$-band interferometric observations obtained with VLTI/MIDI aimed at spatially resolving the mid-infrared emitting region of the disk. We will also compare the thermal coupling between dust and gas in the disks surroundings our three targets by comparing the MIDI observations to a simple phenomenological model derived from the [O I] data. The properties of the programme stars are reported in Table 1. The paper is organized as follow: Section 2 briefly summarizes the results of Paper I; Sect. 3 contains information about observations and data reduction; in Sects. 4 and 5 we report respectively an analysis of the interferometric measurements and the comparison with the optical data of Paper I. The discussion and conclusion are given in Sect. 6.

\section{2. [O I] emission: Paper I}

The [OI] $6300 \AA$ emission in young intermediate-mass stars is thought to be caused by photodissociation of the $\mathrm{OH}$ molecules present in the protoplanetary disk by stellar UV photons. A fraction of the resulting excited oxygen atoms is in the upper state $\left({ }^{1} D_{2}\right)$ of the $6300 \AA$ transition. The [OI] $6300 \AA$ line is thus non-thermal and strongly sensitive to the UV radiation from the central star (Acke et al. 2005). For this reason, the oxygen line traces only the surface layers of the disk that are directly exposed to the stellar radiation field.

The Doppler broadened oxygen emission profile was translated into an amount of emission as a function of distance from the central star assuming Keplerian rotation of the gas. The resulting radial profile of the [O I] emission is in agreement with the expected disk shapes as derived from their spectral energy distribution (SED) according to the phenomenological classification of Meeus et al. (2001). For all targets the oxygen emission starts at velocities corresponding to their dust sublimation radius and extends up to radii of 10-90 AU. The [O I] radial profile shows a double peak structure in the case of HD 101412 and HD $135344 \mathrm{~B}$, with a first stronger peak at $\sim 1 \mathrm{AU}$, and a second weaker peak at 5-10 AU. In HD 179218 the [O I] has a single peak at $\sim 3-6$ AU. The normalized intensity [O I] radial profiles for the three stars are shown in Figs. 8-10.

\section{Observations and data reduction}

MIDI $^{1}$ (Leinert et al. 2003) is the mid-infrared ( $\left.8-13 \mu \mathrm{m}\right)$ beam combiner of the VLT interferometer on Cerro Paranal, Chile. MIDI can combine the light of any pair of the 4 ESO VLT telescopes (UT, 8.2-m). MIDI allows spectrally resolved observations, that is, the signal is dispersed by either a prism $(\Delta \lambda / \lambda \approx$ 30 ) or a grism $(\Delta \lambda / \lambda \approx 300)$. For our project we used the low resolution (prism) mode and two nearly perpendicular baselines: UT1-UT2 $\left(B=57 \mathrm{~m}, \mathrm{PA}=26^{\circ}\right)$ and UT3-UT4 $(B=62 \mathrm{~m}$, $\mathrm{PA}=111^{\circ}$ ). Taking advantage of the earth rotation (which modifies the projection of the baseline on the sky) we observed our targets at different sidereal times, to optimize the coverage of the $(u, v)$ plane.

Two modes are available for the acquisition of the interferometric signal: "High Sens" and "Sci Phot". With the first mode, the photometric signal (i.e. the pure, not combined, $8-13 \mu \mathrm{m}$ spectrum of the source coming from the two telescopes) is acquired soon after the interferometric signal. In Sci Phot mode, the two signals are acquired at the same time. We used both modes depending on the $N$-band luminosity of the source. The $\log$ of MIDI observations is reported in Table 2. The data were reduced with the standard data reduction software MIA+EWS v1.5.2 2,3 using the Expert Work Station (EWS). Detailed documentation on the reduction procedure can be found in Ratzka (2005) and Jaffe (2004) or in the cited web pages.

Standard stars (HD 107446, HD 129456 and HD 188512) were observed close in time and in airmass to the science targets. The angular diameter of these stars is well known and they can be used as calibrators for the transfer function of the instrument. Other calibrators observed during these nights were used

\footnotetext{
1 wWW.eso.org/instruments/midi

2 wWW.mpia-hd.mpg.de/MIDISOFT

3 wWW.strw. leidenuniv.nl/ koehler/MIA+EWS-Manual
} 
Table 2. Summary of VLTI/MIDI observations.

\begin{tabular}{|c|c|c|c|c|c|c|c|c|}
\hline Night & $\begin{array}{l}\text { UT } \\
\text { [hh:mm] }\end{array}$ & "Mode & $\overline{\bar{\lambda} \lambda / \Delta \lambda}$ & Baseline & $\begin{array}{l}B_{\perp} \\
{[\mathrm{m}]} \\
\end{array}$ & $\begin{array}{l}\text { PA } \\
{\left[{ }^{\circ}\right]} \\
\end{array}$ & Airmass & "Calibrator \\
\hline \multicolumn{9}{|c|}{ HD 101412} \\
\hline 2006-05-15 & $23: 38-00: 02$ & SP & 30 & UT3-UT4 & 59 & 102 & 1.2 & HD 107446 \\
\hline 2006-05-16 & $01: 55-02: 18$ & SP & 30 & UT3-UT4 & 62 & 110 & 1.3 & HD 107446 \\
\hline 2006-06-13 & $00: 18-00: 34$ & SP & 30 & UT1-UT2 & 43 & 30 & 1.3 & HD 107446 \\
\hline \multicolumn{9}{|c|}{ HD 135344 B } \\
\hline 2006-05-13 & $04: 32-05: 15$ & HS & 30 & UT3-UT4 & 62 & 112 & 1.0 & HD 129456 \\
\hline $2006-$ & $23: 26-23: 52$ & HS & 30 & UT1-UT2 & 56 & 27 & 1.3 & 29456 \\
\hline 2006-07-12 & $03: 14-03: 47$ & HS & 30 & UT1-UT2 & 47 & 10 & 1.3 & HD 129456 \\
\hline \multicolumn{9}{|c|}{ HD 179218} \\
\hline 2006-05-16 & $05: 25-05: 44$ & SP & 30 & UT3-UT4 & 58 & 128 & 1.7 & 88512 \\
\hline 2006-06-13 & $06: 42-06: 56$ & SP & 30 & UT1-UT2 & 48 & 42 & 1.3 & HD 188512 \\
\hline 2006-06-13 & $08: 07-08: 25$ & SP & 30 & UT1-UT2 & 53 & 40 & 1.5 & HD 188512 \\
\hline 2006-07-08 & $06: 45-07: 03$ & SP & 30 & UT3-UT4 & 44 & 84 & 1.6 & HD 188512 \\
\hline
\end{tabular}

Column "Mode" indicates the beam combination mode of the interferometric signal: High-Sens (HS) or Sci-Phot (SP). Columns " $B_{\perp}$ " and "PA" list the length of the projected baseline and its position angle (east of north).

to check the stability of the transfer function. The error on the calibrated visibility of the science targets is the standard deviation resulting from calibration with different calibrator stars for each night. Figure 1 shows the transfer function (i.e. the instrumental visibility of the calibrators) for the six nights of observations. For each night, all the calibrators observed with the prism in both modes (HS and SP) are plotted. The overall shape of the transfer function with wavelength varies by less than $10 \%$ during the night. The standard stars were also used to flux calibrate the mid-infrared spectrum of the science targets. Conversion factors from counts to Jy were computed from theoretical spectral energy distributions (SED). Energy distributions were derived by matching the spectral type of the calibrators to stars in the Cohen list (Cohen et al. 1999) and scaling the spectrum with the ratio of their IRAS $12 \mu \mathrm{m}$ fluxes.

The averaged MIDI spectra of the three targets are shown in Fig. 2. The Spitzer spectrum is plotted for comparison. Each spectrum was scaled in order to match the Spitzer spectrum at $9 \mu \mathrm{m}$. MIDI and Spitzer spectra agree well. A systematic deviation of the MIDI spectrum from the Spitzer one is visible at the red edge of the $N$-band. This is due to a non-perfect correction of the MIDI spectrum for atmospheric absorption.

\subsection{Note on the data reduction of HD $135344 B$}

The data reduction process of HD $135344 \mathrm{~B}$ deserves a more detailed description. The brightness of this target is close to the sensitivity limit of the instrument. To check the quality of the data we used both the coherent (EWS) and incoherent (MIA) method. Given the faintness of the source, we used a higher threshold for the "good scans" in MIA (60-70\%). In all cases, small differences at the level of $10-20 \%$ in the calibrated visibility are present. In particular, on the night of 2006 July 12, EWS yields a lower visibility at short wavelengths. After different checks, the problem seems to be the fixed mask used to extract the signals with EWS. Both the bi-dimensional interferometric and photometric signal are slightly offset compared to the position of the EWS mask. For this reason we preferred to adopt the mask computed by MIA. With this procedure the two data reduction packages produce very similar results which are consistent with a precision of $\lesssim 10 \%$.

\section{Data analysis}

The quantity measured by an interferometer is the Fourier transform of the brightness distribution, i.e. the complex visibility. Due to atmospheric turbulence, however, the phase of this complex number is corrupted and cannot be retrieved. The amplitude of the complex visibility - the visibility - can be measured and is compared to the corresponding quantity of theoretical brightness distributions. The visibility is a two-dimensional function in the so-called $u, v$-plane, where $u$ and $v$ are the spatial frequencies $B / \lambda$ ( $B$ is the baseline length between the two telescopes, and $\lambda$ the wavelength) in two perpendicular directions. In the case of VLTI/MIDI, a single observation covers a range of spatial frequencies due to the spectral capability of the beam combiner. However, one should keep in mind that the brightness distribution of the target may vary from wavelength to wavelength.

Figures 3, 4 and 6 show the calibrated MIDI visibilities of HD 101412, HD 135344 B and HD 179218 respectively. We plot the visibility versus spatial frequency rather than wavelength, in order to make a direct comparison with the spatial distribution of the [O I] emission measured at $6300 \AA$. For convenience, we plot the MIDI wavelength scale at the top of the figure. The slope of the visibility curve in the $N$-band depends on: 1 ) the increase in spatial resolution with decreasing wavelength; and 2) the wavelength-dependence of the brightness distribution.

The [O I] visibility curves (dashed line) are calculated from the radial intensity profile presented in Paper I and are discussed later in the text. The comparison of MIDI observations and [O I] visibility is presented in Sect. 5. In this section we use a simple geometrical model aimed at deriving the geometrical properties (inner and outer radius, inclination and position angle) of the (mid-infrared) dust emitting region in the three protoplanetary disks.

\subsection{Uniform ring model}

In a first approximation the brightness distribution of a protoplanetary disk might be described by a uniform bright ring (UR). The formalism of the UR model is described in Appendix B. We emphasize that the UR model is a simple geometrical approximation of the dust emitting region. This model does not take into account the physical properties of the circumstellar disk (e.g. dust sublimation radius, disk outer radius, temperature 


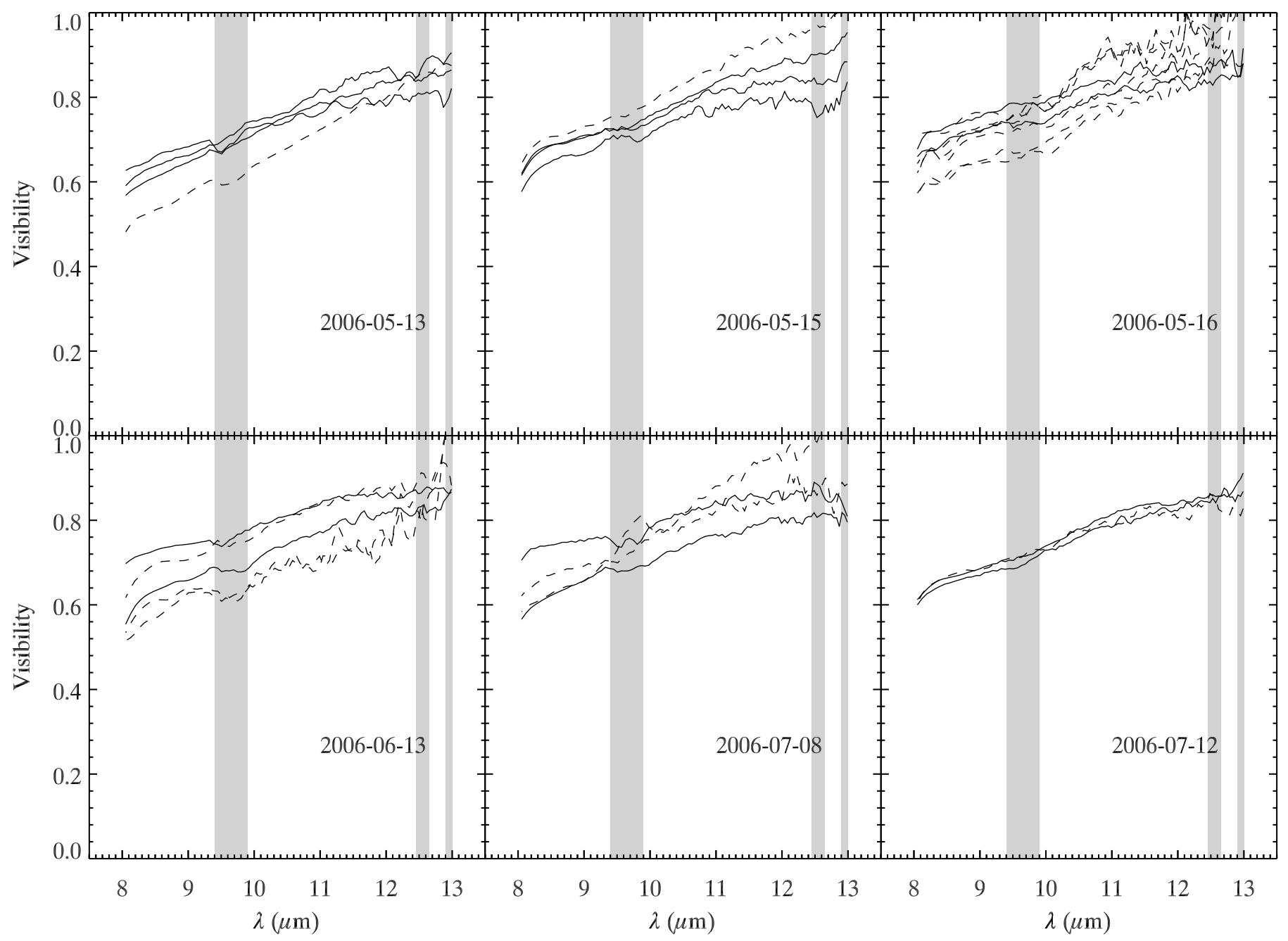

Fig. 1. Transfer function of VLTI/MIDI. For each night, the different lines correspond to different calibrators observed with the prism in both modes: HS (solid line) and SP (dashed line). The overall shape of the transfer function, for a given mode, does not drastically change during the night. The vertical grey regions are characterized by strong atmospheric absorption bands. Residuals of the data reduction may be present within these regions.

gradient) or the dust properties (e.g. grain size). Such a model has been extensively used as an analytic model for nearinfrared interferometric observations of disks around young stars (e.g. Millan-Gabet et al. 2001; Eisner et al. 2004, 2007) as well as mid-infrared interferometric data of planetary nebulae (Chesneau et al. 2006). The MIDI visibilities were fitted using an inclined uniform ring (UR) model.

We have searched for the best set of model parameters by minimizing the $\chi^{2}$ of the visibility measurements. The values of the best fit parameters and associated errors were computed using a Monte Carlo simulation. Assuming a normal error distribution of the measured visibility, we simulated 100 random data-sets around the observed values. The best fit parameters and errors correspond to the mean and the standard deviation of the 100 fits.

\section{2. $H D 101412$}

HD 101412 (Fig. 3) is barely resolved at all epochs. Along the three baseline position angles the visibility is lower at short wavelengths and increases from $8 \mu \mathrm{m}$ to $9-10 \mu \mathrm{m}$. Differences in the absolute value and shape in the three measurements may suggest an asymmetric emission. In particular, HD 101412 is more resolved (i.e. shows a more extended emission) with the shortest baseline, $43 \mathrm{~m}$ at $30^{\circ}$. Along this direction the visibility goes from 0.55 at $8 \mu \mathrm{m}$ to 0.9 at $13 \mu \mathrm{m}$. Around $11.3 \mu \mathrm{m}$ the disk appears larger than at adjacent wavelengths (with a small dip in visibility). This coincides with PAH emission. The high visibility values suggest a compact (mid-infrared) dust emission. The decreasing visibility with decreasing wavelength is due to the increase in spatial resolution. The three visibility measurements of HD 101412 are best fitted by a UR model (Fig. 3, solid line) of the inner and outer radius of 0.4 and $1.9 \mathrm{AU}$, an inclination of $80^{\circ}$ and position angle of the disk major axis of $38^{\circ}$. The almost edge-on orientation found here explains why the [O I] line profile shows structures that are not seen in other [O I] profiles of Herbig stars; thanks to the high inclination, the projected velocities are close to the real velocities, and the Dopplerinduced spread in the spectrum is optimal.

According to the classification of Meeus et al. (2001), the SED of HD 101412 suggests a self-shadowed disk geometry. In this case the mid-infrared disk emission is expected to arise only from the inner rim of the disk. This is consistent with the small size measured with MIDI. 


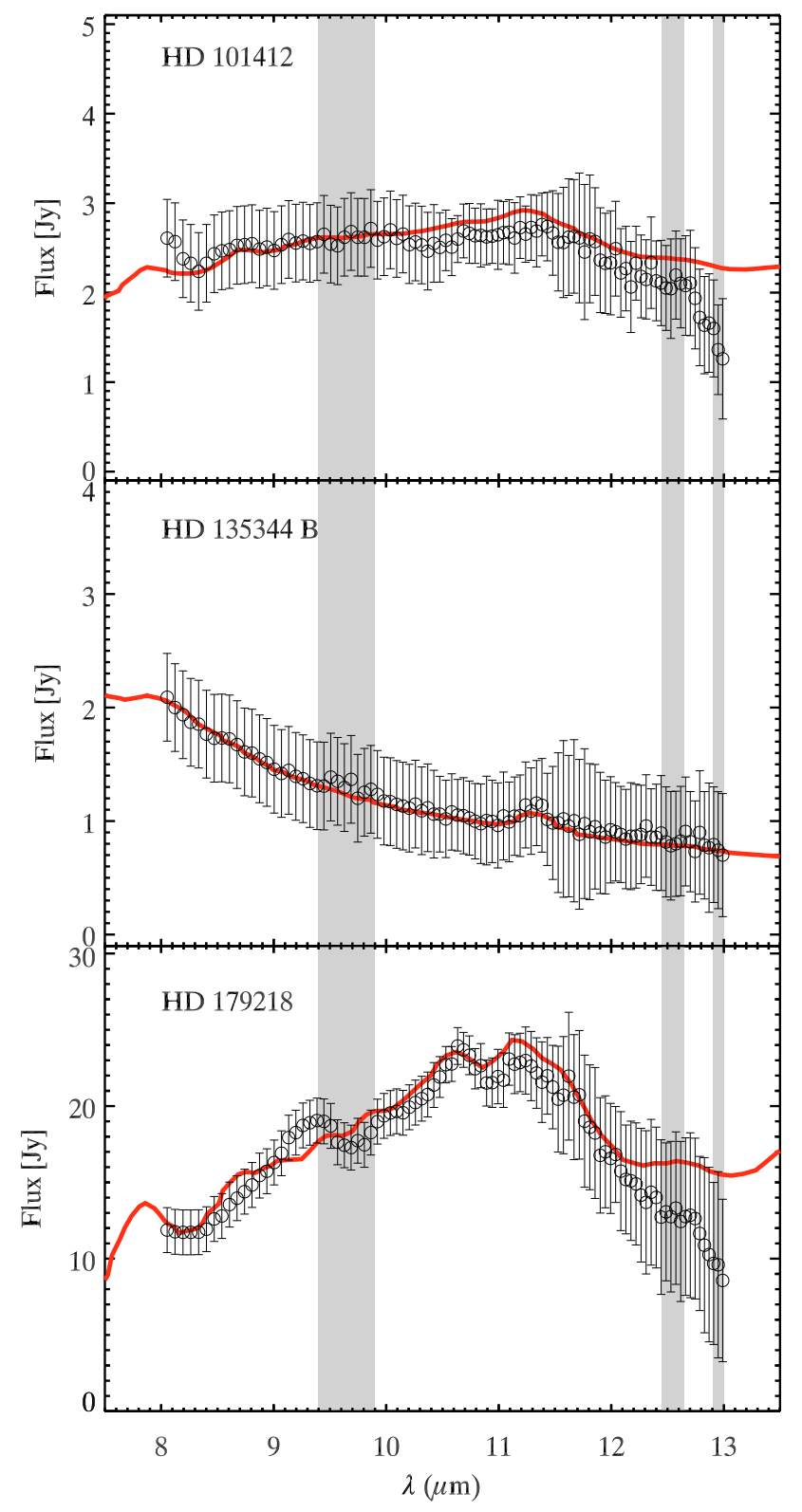

Fig. 2. MIDI spectra (diamonds) of HD 101412, HD 135344 B and HD 179218. The MIDI spectra were scaled to match the Spitzer IRS spectra (red line) at $9 \mu \mathrm{m}$.

\section{3. $H D$ 135344B}

HD $135344 \mathrm{~B}$ is resolved with all baselines (Fig. 4). Along the $47 \mathrm{~m}$ baseline (position angle $10^{\circ}$ ) the visibility decreases with wavelength. Along the second and third baselines, $56 \mathrm{~m}$ and $62 \mathrm{~m}$ respectively, the visibility shows a similar shape: nearly constant between $8-11 \mu \mathrm{m}$ with a small bump around 9.2-9.4 $\mu \mathrm{m}$, a drop at $11.3 \mu \mathrm{m}$ and almost constant at longer wavelength. Despite the higher resolution, the source is less resolved at short wavelengths. This means that at longer wavelengths the target is significantly more extended due to emission of the colder outer disk which only starts to show up at these wavelength. This specific pattern is sometimes observed in mid-infrared interferometric observations of young PMS stars (e.g. V1647 Orionis, Abraham et al. 2006; FU Orionis, Quanz et al. 2006). Reasonable fitting of this visibility shape is obtained with a (physical) Keplerian, flared disk model where temperature and surface density are prescribed by a broken power
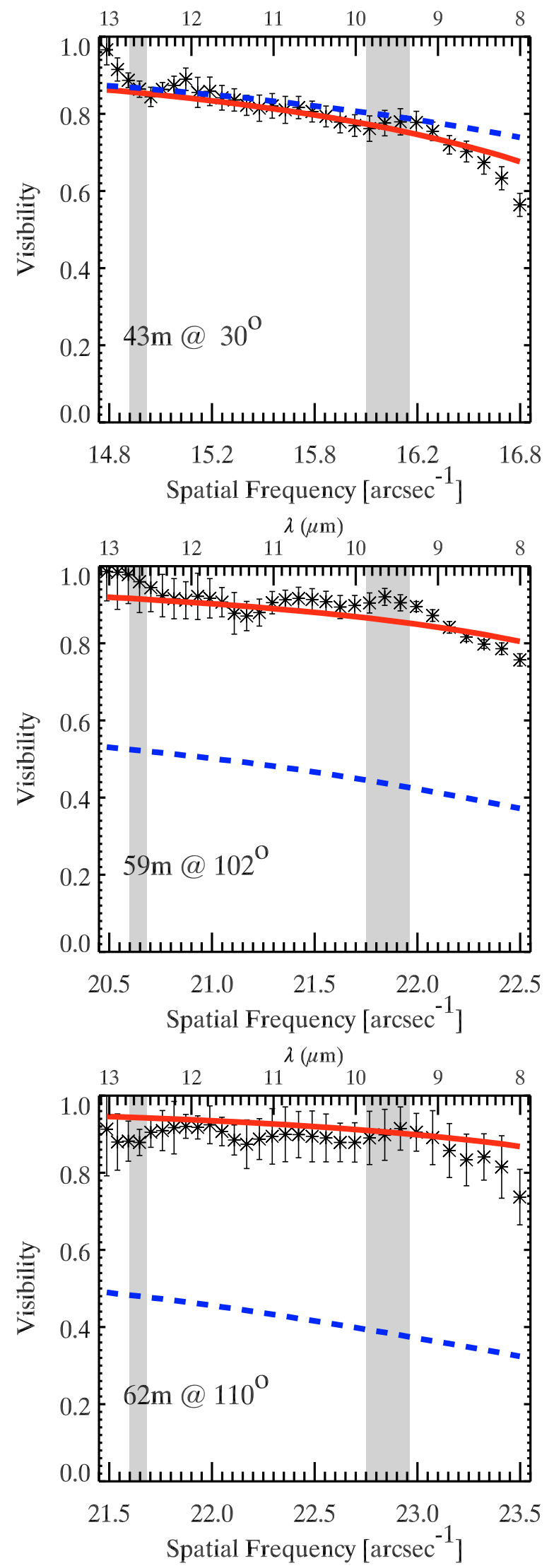

Fig. 3. Calibrated visibility amplitude at different baselines for HD 101412. The dashed line in the visibility panel represents the visibility computed from the intensity versus radius profile of the [O I] $6300 \AA$ A emission line. The solid line is the best fit of the inclined UR model. Visibility errors are computed as standard deviations resulting from calibrations with different calibrator stars. 

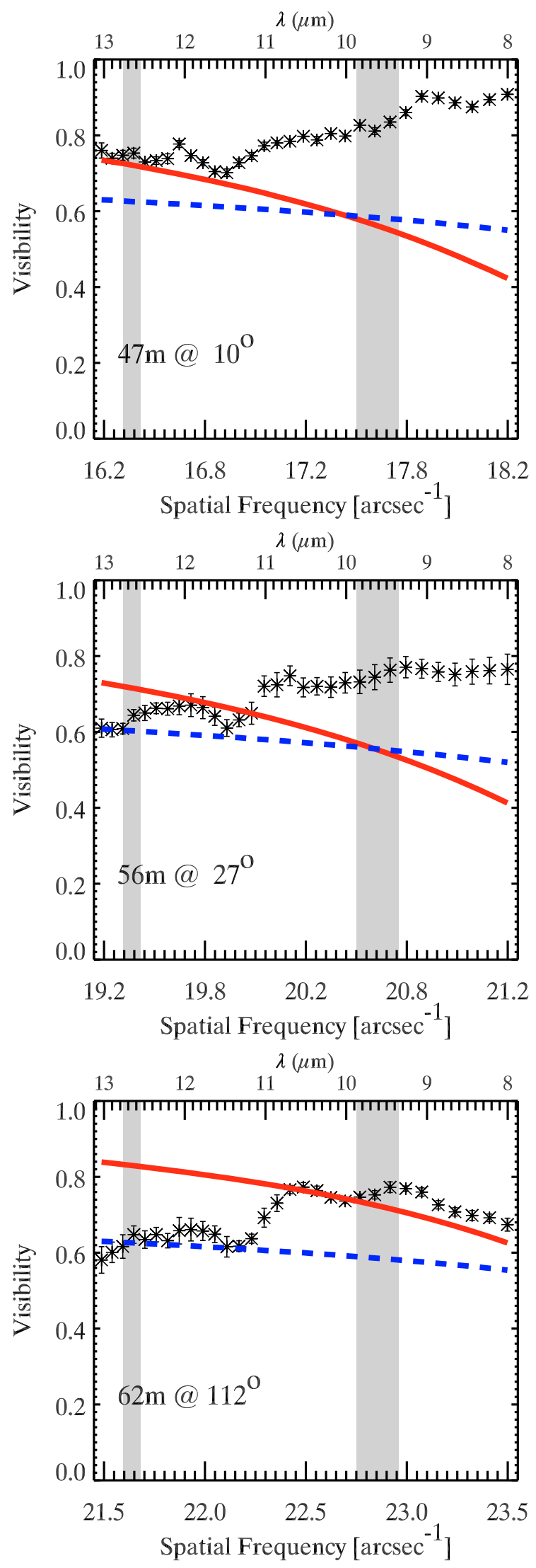

Fig. 4. Same as Fig. 3 for HD 135344 B.

law distribution $\left(T \propto r^{-q} ; \Sigma \propto r^{-p}\right)$, i.e. the disk's surface is cooler at larger radii. The result of the UR model (Fig. 4, solid line and Table 3) are: inner radius $0.05 \mathrm{AU}$, outer radius $1.8 \mathrm{AU}$

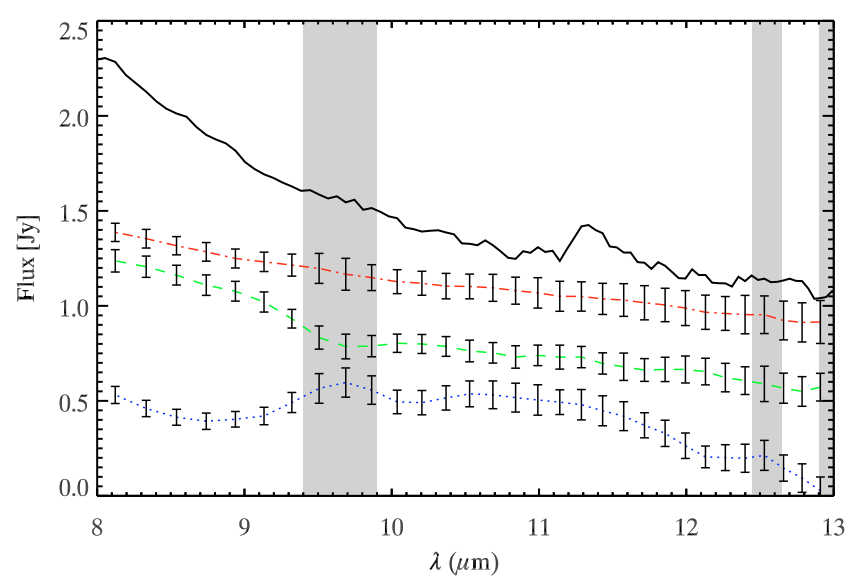

Fig. 5. Correlated spectrum of HD 135344 B measured on 2006 May 13 (dotted), 2006 June 13 (dashed) and 2006 July 12 (dot-dashed). The spectra were shifted for clarity. The mean, single telescope, spectrum is also plotted (solid line). The absence of the PAH emission feature at $11.3 \mu \mathrm{m}$ in the correlated spectrum is a signature of spatially extended PAH emission compared to the continuum emission.

Table 3. Best fit parameters of the UR model for HD 101412 and HD 135344 B.

\begin{tabular}{llllll}
\hline \hline Star & $\begin{array}{l}R_{\text {in }} \\
{[\mathrm{mas} / \mathrm{AU}]}\end{array}$ & $\begin{array}{l}R_{\text {out }} \\
{[\mathrm{mas} / \mathrm{AU}]}\end{array}$ & $\begin{array}{l}i \\
{\left[{ }^{\circ}\right]}\end{array}$ & $\begin{array}{l}\phi \\
{\left[{ }^{\circ}\right]}\end{array}$ & $\tilde{\chi}^{2}$ \\
\hline HD 101412 & $2.5 \pm 0.6$ & $11.9 \pm 0.6$ & $80 \pm 7$ & $38 \pm 5$ & 0.4 \\
& $0.4 \pm 0.1$ & $1.9 \pm 0.1$ & & & \\
HD 135344 & $0.35 \pm 1.79$ & $12.8 \pm 1.4$ & $60 \pm 10$ & $180 \pm 60$ & 3.9 \\
& $0.05 \pm 0.25$ & $1.8 \pm 0.2$ & & & \\
\hline
\end{tabular}

$R_{\text {in }}$ and $R_{\text {out }}$ are the inner and outer ring radii and are given in milliarcsec (first row) and AU (second row). The last column lists the value of the reduced $\chi^{2}$. Values close to one indicate a good agreement between model and observations.

(assuming a distance of $140 \mathrm{pc}$, van Boekel et al. 2005), inclination $60^{\circ}$ and position angle of the disk major axis $180^{\circ}$. The inclination found here is slightly higher than the result of Doucet et al. (2006) $\left(45^{\circ}\right)$, obtained with direct imaging at $20.5 \mu \mathrm{m}$. Dent et al. (2005), modeling the $J=3-2$ transition of ${ }^{12} \mathrm{CO}$, find a smaller disk inclination $\left(11^{\circ}\right.$, disk nearly face-on). Nevertheless, the UR model is not able to reproduce the decreasing visibility observed with MIDI and the disk parameters are not well constrained.

We converted the MIDI visibility to the Gaussian full width half maximum $(F W H M)$ at each spatial frequency. The $F W H M$ represents the extent of the emitting region and is defined as:

$\theta=\sqrt{\frac{\ln (V(s f))}{-3.56 s f^{2}}}$

where $V(s f)$ is the MIDI visibility at spatial frequency $s f$. A Gaussian distribution is only a crude approximation of the real brightness distribution valid at high visibilities. Similarly to Quanz et al. (2006), we also fitted the FWHM measured on each baseline with an ellipse wavelength by wavelength. The best-fit ellipse parameters at three reference wavelengths $(9 \mu \mathrm{m}$, $11 \mu \mathrm{m}, 12.5 \mu \mathrm{m})$ are listed in Table 4. As expected, the disk looks larger at longer wavelengths. The ellipse semi-major axis increases from $1.1 \mathrm{AU}$ at $9 \mu \mathrm{m}$ to $1.9 \mathrm{AU}$ at $12.5 \mu \mathrm{m}$. The inclination and position angle varies by $\sim 5^{\circ}$ and $\sim 9^{\circ}$ respectively 

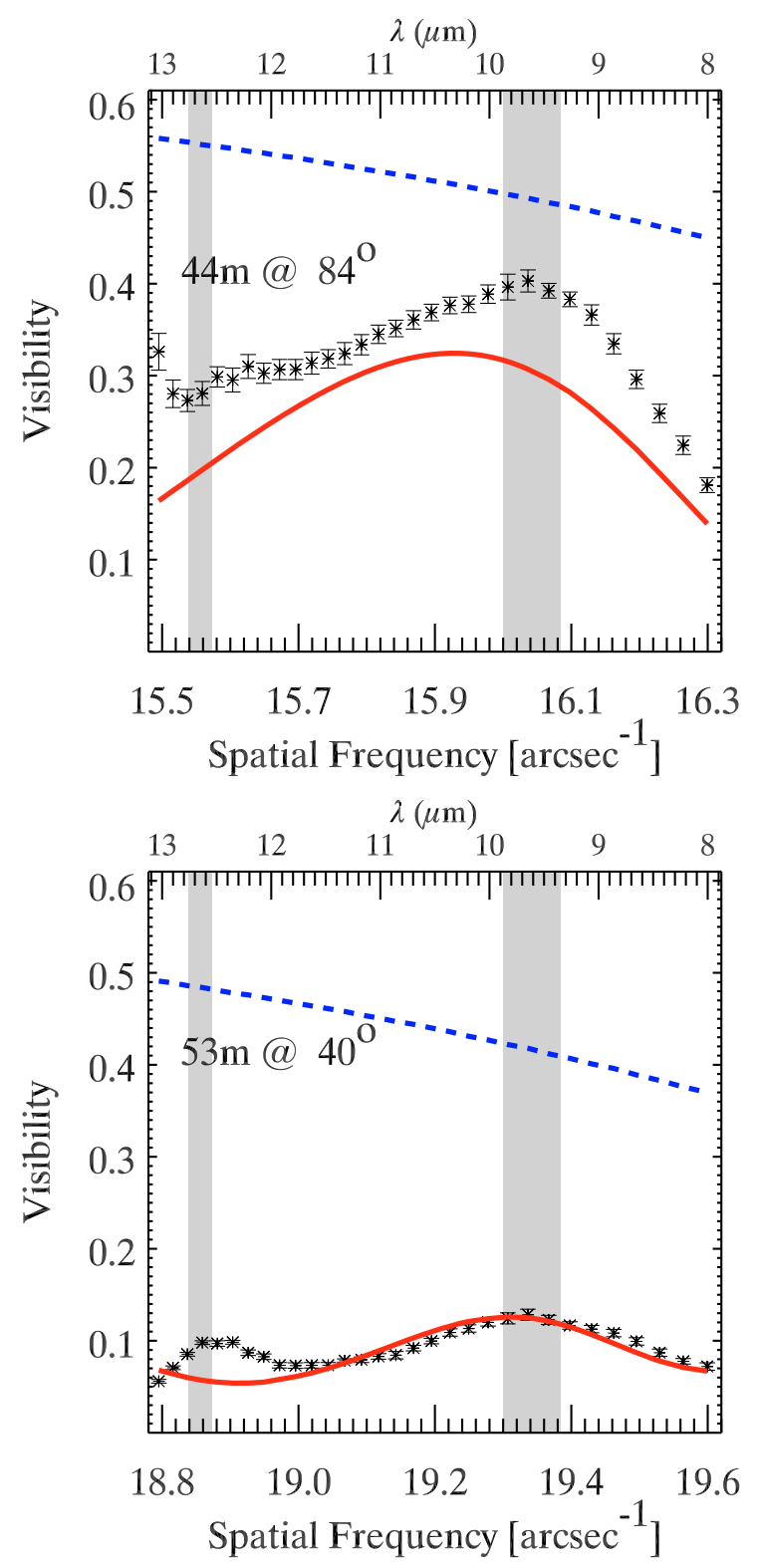
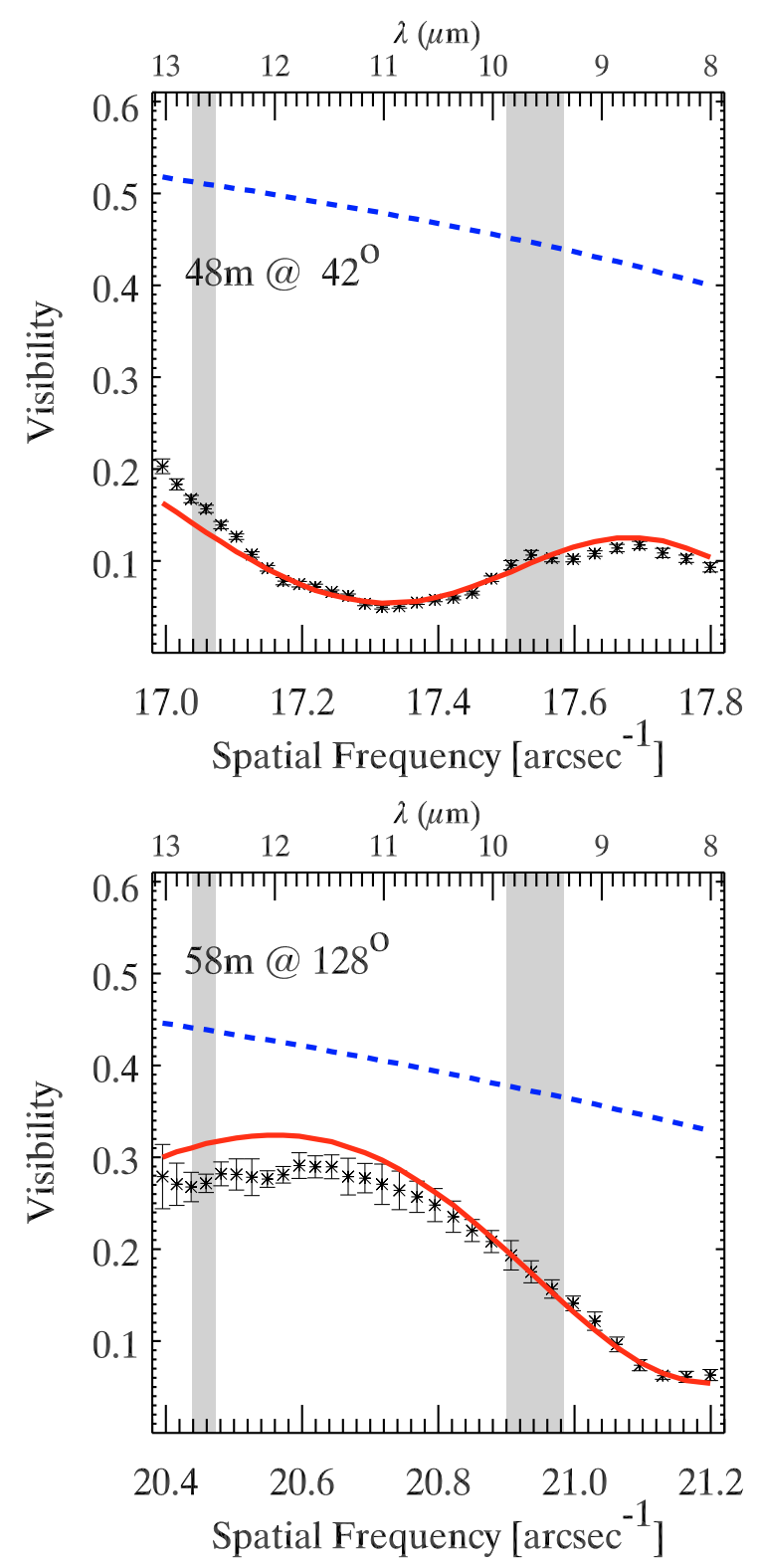

Fig. 6. Same as Fig. 3 for HD 179218. The continuous line here represents the best fit two-component disk model.

Table 4. Best-fit ellipse parameters for HD 135344 B.

\begin{tabular}{llll}
\hline \hline Wavelength & $9 \mu \mathrm{m}$ & $11 \mu \mathrm{m}$ & $12.5 \mu \mathrm{m}$ \\
\hline Semimajor axis [AU] & 1.1 & 1.5 & 1.9 \\
Semiminor axis [AU] & 0.6 & 0.8 & 0.9 \\
Inclination $\left[{ }^{\circ}{ }^{\text {] }}\right.$ & 53 & 58 & 61 \\
Position angle $^{\circ}{ }^{\circ}$ ] & 171 & 163 & 163 \\
\hline
\end{tabular}

The ellipse is fitted to the $F W H M$ measured on the three baselines. Ellipse axis are computed assuming a distance of $140 \mathrm{pc}$.

from $9 \mu \mathrm{m}$ to $11 \mu \mathrm{m}$. The $11 \mu \mathrm{m}$ and $12.5 \mu \mathrm{m}$ ellipses have similar (within $\sim 3^{\circ}$ ) inclinations and position angles. The geometry of the mid-infrared dust emission derived with the UR model and the ellipse fitting are consistent with each other.

The SED of HD 135344 B is characterized by a relatively low infrared excess over the stellar photosphere up to $13 \mu \mathrm{m}$ and by a strong infrared excess beyond $14 \mu \mathrm{m}$. Moreover, the mid-infrared spectrum (Fig. 2) lacks silicate features and only weak PAH emission is detected. Such an SED is peculiar among group I sources and suggests a more complex disk structure. The missing near- to mid-infrared excess and the fast increase at longer wavelengths have been attributed to the presence of a large dust-free gap by Brown et al. (2007). They proposed a so-called "cold disk" structure for the material around HD 135344 B. In their model the disk is devoid of dust between 0.45-45 AU (in radius). According to Brown et al., warm dust is present in an inner ring between 0.18-0.45 AU. The MIDI observations presented here are consistent with this model. The Gaussian FWHM, if scaled to the distance adopted by Brown et al., yields an emitting region between 0.4-0.7 AU. Although these radii are larger than those found by Brown et al. (note that we used a simple Gaussian model), the two estimates agree within the uncertainty of the data and the analysis.

Because MIDI is only sensitive to dust emitting at $10 \mu \mathrm{m}$, our data set does not allow us to claim the presence or absence of cold matter beyond the inner warm disk. The shadow of the inner disk may obscure a large portion of the disk which re-emerges only at large radii. Both the SED and MIDI observations are only sensitive to the dust emitting in the mid-infrared, both the SED 
and our MIDI observations are not able to distinguish between a (partially) self-shadowed disk and a disk with a gap.

The correlated spectrum of HD 135344 B for the different measurements is plotted in Fig. 5. At all epochs the correlated spectrum mimics the total (single-telescope) spectrum, although deviations are visible. The $11.3 \mu \mathrm{m}$ PAH emission detected in the total spectrum is not present in any of the correlated spectra which indicates that the PAH $11.3 \mu \mathrm{m}$ emission region is completely resolved at the angular resolution of our measurements.

\section{4. $H D 179218$}

Despite being the most distant object of our sample, HD 179218 is the most spatially resolved source of the three objects. The visibility varies with the position angle of the baseline and is always below 0.4 . Along the first baselines the visibility increases rapidly from 8 to $9 \mu \mathrm{m}$ and decreases afterwards. Along the second and third baselines (48 and $53 \mathrm{~m}$ ), the visibility is much lower and shows a characteristic sinusoidal modulation. In the last case, the visibility increases from a very low value, 0.05 at $8 \mu \mathrm{m}$ to 0.27 at $11.7 \mu \mathrm{m}$ and decreases afterwards.

A sinusoidal pattern of visibility is produced if the brightness distribution of the target displays sharp edges, e.g. in the case of a ring, uniform disk or a binary with two unresolved components. A smooth emission region, such as a Gaussian disk, would not produce such variations. We can immediately exclude the binary hypothesis: the MIDI observations of HD 179218 cover a range in position angles over $90^{\circ}$ and the visibility always shows a sinusoidal pattern. In the case of a binary, the pattern is PA dependent and completely disappears when the PA of the baseline and that of the binary axis are aligned. We attempted to fit the MIDI visibility with the UR model but although this model is able to reproduce the sinusoidal modulation of the visibility, it fails to reproduce the visibility at the position of the local "minima". The minimum in the ring model is zero while the observed visibility reaches a minimum value of $V=0.05$. If these were "true" zeroes of the visibility, i.e. the location between two adjacent lobes of the visibility curve, we would have measured a contrast of $V \sim 0.0076^{4}$. We then attempted to fit the data with a two-component model. The first component represents the disk emission at small radii and the second component represents the disk surface at larger radii. Our simulations showed that the first component is only barely resolved. We used a uniform ring for both the internal and external component. Using a different brightness distribution for the internal component does not improve the model fit. The fractional flux contribution of the first component, $f_{\text {int }}$, was left as a free parameter in the fit. For simplicity, $f_{\text {int }}$, was assumed to be constant at all wavelengths. The best fit parameters are listed in Table 5. Inclination and position angle are in agreement with those found with the single ring model $\left(i=57^{\circ}, \mathrm{PA}=198^{\circ}\right)$. The inner component contributes $20 \%$ of the total mid-infrared flux. According to this model, the disk around HD 179218 is either truncated or obscured by its own shadow between 3.2 and 14.5 AU radii from the star (Table 5).

Previous infrared interferometric observations of HD 179218 have been carried out by by Leinert et al. (2004) and Liu et al. (2007) in the mid-infrared and by Monnier et al. (2006) in the $H$-band. Leinert et al. also measured low visibilities with an $80 \mathrm{~m}$ baseline. Monnier et al. (2006) measured

\footnotetext{
4 This value, given the finite band-width of the MIDI wavelength bins, corresponds to the visibility null reachable by the interferometer at low spectral resolution (PRISM).
}

Table 5. Best fit parameters and standard deviation of the twocomponent disk model for HD 179218.

\begin{tabular}{ll}
\hline \hline HD 179218 & \\
\hline 1st component: $R_{\text {in }}(\mathrm{AU})$ & $0.3 \pm 0.1$ \\
1st component: $R_{\text {out }}(\mathrm{AU})$ & $3.2 \pm 0.1$ \\
2nd component: $R_{\text {in }}(\mathrm{AU})$ & $14.5 \pm 0.5$ \\
2nd component: $R_{\text {out }}(\mathrm{AU})$ & $22.6 \pm 0.4$ \\
inclination $\left({ }^{\circ}\right)$ & $57 \pm 2$ \\
PA $\left({ }^{\circ}\right)$ & $23 \pm 3$ \\
$f_{\text {in }}$ & $0.2 \pm 0.02$ \\
\hline
\end{tabular}

Radii are given in AU assuming a distance of 240 pc. The inclination and position angle of the two components were assumed to be equal for both structures. $f_{\text {in }}$ is the fractional contribution of the inner component to the total flux in the mid-infrared.

a squared $H$-band visibility of the order of $40 \%$, corresponding to a Gaussian FWHM of 3.8 AU. This is in good agreement with our two-component model, where the inner component extends up to 3.5 AU from the star. Liu et al. modeled MMT nulling interferometric data by means of a ring of diameter $27 \pm 5 \mathrm{AU}$. This large ring is, within the uncertainties, consistent with the outer ring of our two-component model.

The agreement between the MIDI data and the simple geometrical model proposed here is quite good. However, deviations from the model are clearly visible. Similarly to HD 135344 B, our observations are not able to distinguish between a real gap (i.e. a region free of material) and a shadowed region.

\subsubsection{Differential phase}

As a byproduct of the EWS data reduction software, the relative phase between the different spectral channels of MIDI can be retrieved. This so-called differential phase is the real differential phase of the target on the sky, modulated by the atmosphere. The largest effect of the latter is an offset of the differential phases, which is linear with respect to wavenumber $\left(\propto \lambda^{-1}\right)$. The EWS software subtracts the best-fit linear component of the measured differential phase, as this information is lost anyway. Secondary atmospheric effects remain: the two light beams, coming from the two telescopes, pass through different amounts of air and water vapor before they reach the MIDI beam combiner. However, these smooth variations in the measured differential phase are of the order of a few degrees. For all targets the differential phase is nearly constant with wavelength and centered at zero degrees. The only exception is the measurements of HD 179218 on 2006 May 16 (Fig. 7). Along this baseline, a large phase variation is detected at short wavelengths. The phase varies by $55^{\circ}$ between 8 and $9 \mu \mathrm{m}$. A flip of $180^{\circ}$ is expected when the visibility amplitude crosses a null, i.e. when the spatial frequencies covered by the observations are located between two adjacent lobes of the visibility curve. In the case of HD 179218 the phase variation is smooth with lower amplitude. The phase variation is then ascribed to a shift of the photo-center of the emitting source. This suggests a deviation from centro-symmetric emission along this direction.

The differential phase plotted in Fig. 7 was not calibrated for the effect of water vapor that is present in the delay line tunnel of the VLT interferometer. The two light beams most likely pass through a different amount of air and water vapor before reaching the MIDI beam combiner. This difference introduces an instrumental differential phase. Figure 7 also shows the differential phase measured with four different calibrators observed during 


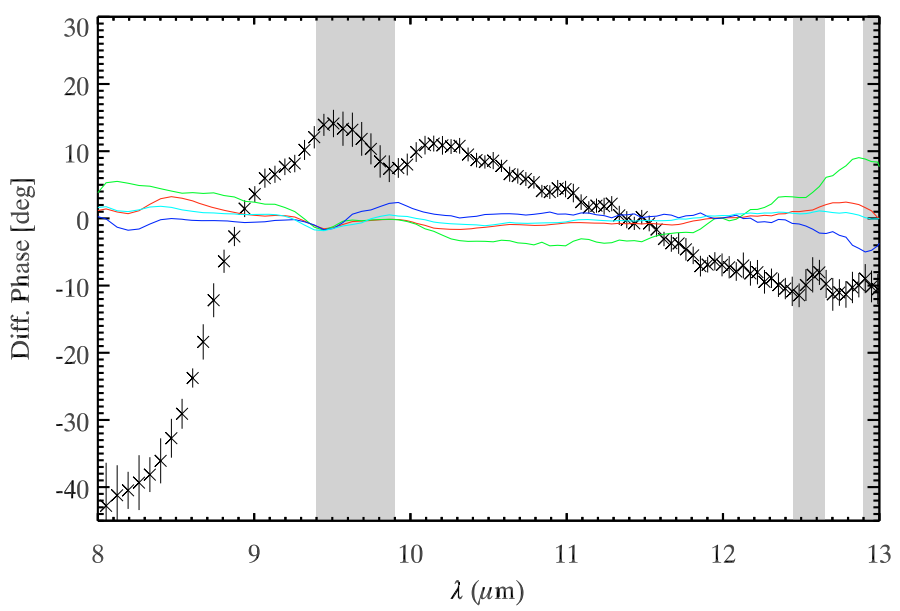

Fig. 7. Differential phase of HD 179218 measured on 2006 May 16. A $55^{\circ}$ phase variation is detected at short wavelengths. The four continuous lines are the differential phases for four different calibrators observed during the same night. The plotted phases are not corrected for the effect of water vapor in the delay lines of the VLTI. See Sect. 4.4.1 for details.

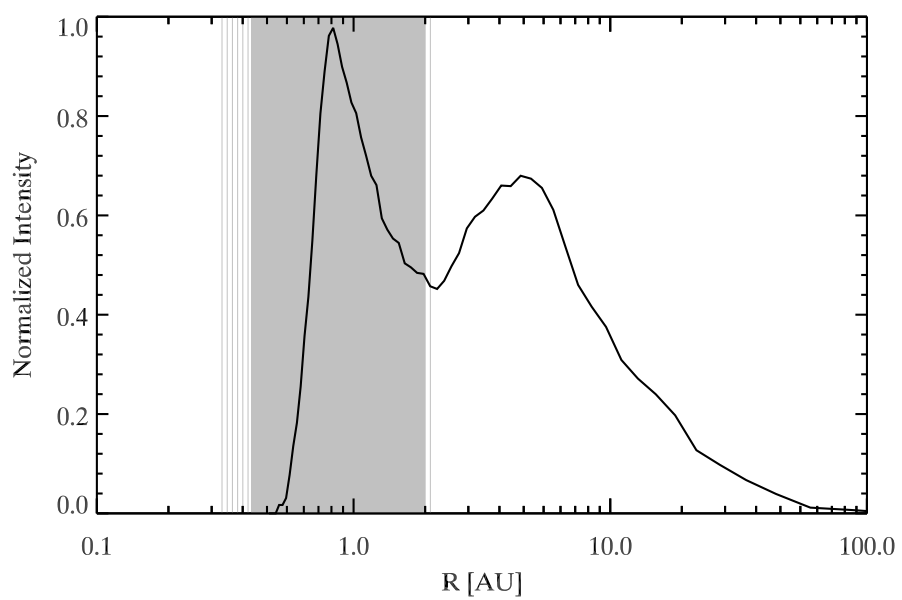

Fig. 8. Normalized [OI] $6300 \AA$ intensity profile for HD 101412. $I_{[\mathrm{O} \mathrm{I}]}(R) \times S(R)$ is the total [O I] line luminosity in a ring at distance $R$ from the star. This was obtained averaging the results of observation $B 1$ and $B 2$ of Paper I. In the specific case of HD 101412, $I_{[\mathrm{O} I]}(R)$ was recomputed with an inclination of $80^{\circ}$. The filled region represents the position of the dust emission region as determined by MIDI. The striped regions indicate the uncertainty on the latter.

the same night with the same acquisition mode (SCI PHOT). Since calibrators are (usually) point-like their differential phase should be zero. The deviation from zero degree in this case is produced by the water vapor. The effect of water vapor is present, but is very small compared to the phase variation observed toward HD 179218.

The variation in the differential phase along the $58 \mathrm{~m}$ baseline is caused by asymmetric emission. This is in agreement with the inclination of the disk $\left(57^{\circ}\right)$ and its flared structure. Due to the flaring, the far side of the disk emits more light in the direction of the observer. This means that the photo-center of this emission (described here by the ring) is no longer coincident with the photo-center of the almost unresolved inner disk. It is difficult to derive a quantitative estimate for this offset, mainly due to the loss of the linear differential phase information. Estimating from the magnitude of the differential phase variation $\left(\sim 60^{\circ}\right)$, it must be around $4 \pm 2$ mas.

\section{Comparison to the optical gas emitting region}

In Paper I very high resolution optical spectra of HD 101412, HD 134355B and HD 179218 were presented. In all three targets, we spectrally resolve the [OI] $6300 \AA$ emission line. Assuming that the disk is in Keplerian rotation, the [O I] emission line profile can be translated into a radial profile, i.e. the intensity-versus-radius curve of the oxygen line emission, $I_{[\mathrm{O} I]}(R)$. Figures $8-10$ show the normalized [O I] radial profile derived in Paper I: $I_{[\mathrm{O} \mathrm{I}]}(R) \times S(R)$, where $S(R)=\pi\left(R_{\text {out }}^{2}-R_{\text {in }}^{2}\right)$ and $R_{\text {out }}$ and $R_{\text {in }}$ outer and inner radius of the ring at radius $R$. $I_{[\mathrm{O}]]}(R) \times S(R)$ is the total luminosity in a ring at distance $R$ from the central star. The [OI] radial profile shown here is based on the average of the spectra presented in Paper I. In the case of HD 101412, $I_{[\mathrm{O} I]}(R)$ was recomputed using a disk inclination of $80^{\circ}$ to be consistent with the result of Sect. 5. Using a larger inclination has the effect of shifting the emission further away from the star as can be seen comparing Fig. 8 with Fig. 11 of Paper I. The filled region represents the position of the dust emission region as determined by MIDI. The striped regions indicate the uncertainty on the latter.

In order to compare the [OI] result with the dust one we need to provide a visibility model for the MIDI observations. From $I_{[\mathrm{O} I]}(R)$, it is possible to derive a brightness distribution $\left(I_{[\mathrm{O} I]}(\alpha, \beta)\right.$, with $\alpha$ and $\beta$ the angular distance from the central star) assuming that the line emission from the disk surface is axis symmetric. Using $I_{[\mathrm{O} I]}(\alpha, \beta)$ as brightness distribution, the visibility model $\left(V_{[\mathrm{O} \mathrm{I}]}(u, v)\right)$ was computed. The expected visibility at the spatial frequencies mapped with MIDI was computed interpolating $V_{[\mathrm{O}]]}(u, v)$ at the corresponding positions in the uv-plane. The dashed line in Figs. 3, 4 and 6 is $V_{[\mathrm{O} I]}(u, v)$ at the spatial frequency covered by MIDI observations.

\subsection{HD 101412}

As shown in Fig. 3, $V_{[\mathrm{O} I]}(u, v)$ is similar to the observed visibility along the first baseline $\left(43 \mathrm{~m}, 30^{\circ}\right)$ and is clearly lower than along the other two directions. This is clearer when looking at Fig. 8. The dust emission is located within $\sim 2 \mathrm{AU}$, while the gas emission appears more extended with a bright peak at 5-6 AU. In terms of radial extent, this means that the [O I] emission extends to larger radii than the mid-infrared emission. Beyond the first peak the [O I] intensity drops off as one would expect for a self-shadowed disk.

\section{2. $H D 135344 B$}

Similarly to HD 101412 the [O I] brightness profile of HD 135344 B shows a double peak with a first stronger peak at small radii $(\sim 0.5 \mathrm{AU})$ and a second fainter peak at $\sim 5-10 \mathrm{AU}$ (Fig. 9). On the other hand, the $10 \mu \mathrm{m}$ dust emission seems to come from the inner 1-2 AU of the disk surface. However, the $[\mathrm{OI}]$ result suffers from overlapping photospheric absorption and thus have to be interpreted with more caution (Paper I). The diagonal striped region in Fig. 9 represents the cold dust detected at longer wavelengths by Doucet et al. (2006) and by Brown et al. (2007).

\section{3. $H D 179218$}

As shown in Fig. 6, the [OI] model is not able to reproduce the observed visibility of HD 179218. The observed visibility is in all cases lower than $V_{\left[\mathrm{O}_{\mathrm{I}}\right]}$. Moreover, the oxygen model increases with wavelength while the observed visibility shows a 


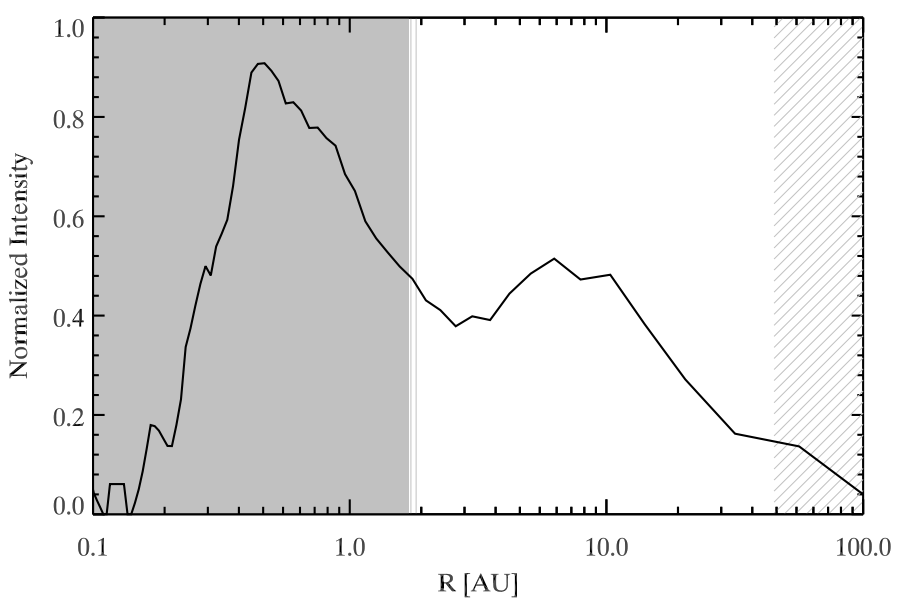

Fig. 9. Same as Fig. 8 for HD 135344 B. The diagonal striped region represents the cold dust in a flared geometry detected by Doucet et al. (2006) and Brown et al. (2007). This cannot be seen with MIDI because it radiates at wavelengths longer than those traced with MIDI.

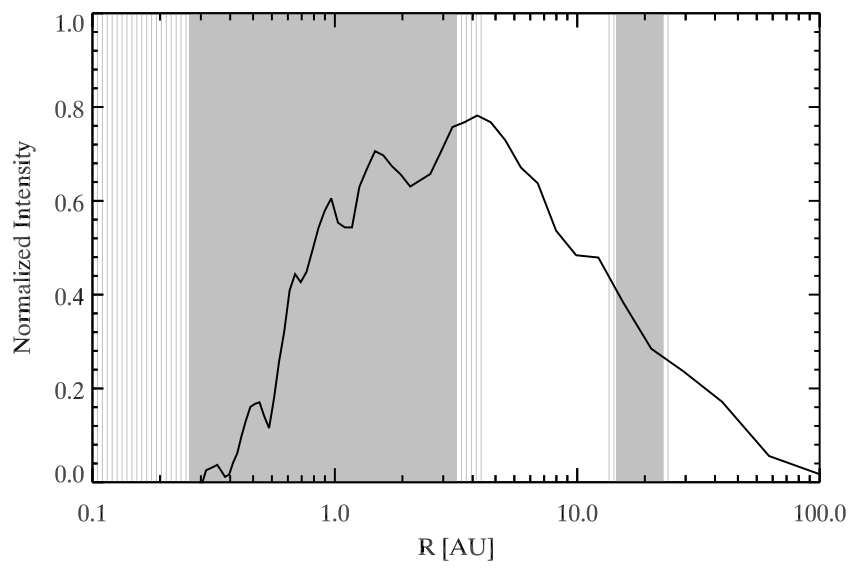

Fig. 10. Same as Fig. 8 for HD 179218.

sinusoidal variation. The $[\mathrm{OI}]$ intensity peaks at 4-6 AU (Fig. 10) and decreases outwards. According to our twocomponent disk model, emitting dust is present within the oxygen peak. A further contribution to the mid-infrared emission, $80 \%$ in terms of flux, comes from a ring located between $\sim 15-$ $23 \mathrm{AU}$. At this distance, the [O I] line intensity is reduced. Our result is consistent with a flared geometry for the disk around HD 179218; the inner, curved, rim is represented by the inner UR while the outer UR may represents the flared disk surface at larger radii. The two dust emission regions are separated by a "dark" (non emitting) region.

\section{Discussion and conclusion}

In this paper we presented the first direct comparison of gas and dust emission from the surface of three protoplanetary disks. The comparison of high optical spectroscopy with infrared interferometric observations gives some insight into the relative size and shape of the gas and the dust emitting region.

For HD 179218 the new MIDI results presented here and the UVES results of Paper I are in good agreement with a flared disk structure. The $10 \mu \mathrm{m}$ emission comes from two separate regions: an inner part located between $\sim 0.3-3$ AU (likely the disk inner rim) and an outer part between $~ 15-23$ AU (flared disk surface). No dust emission is detected between 3-15 AU. The [O I] brightness profile shows instead a single strong peak
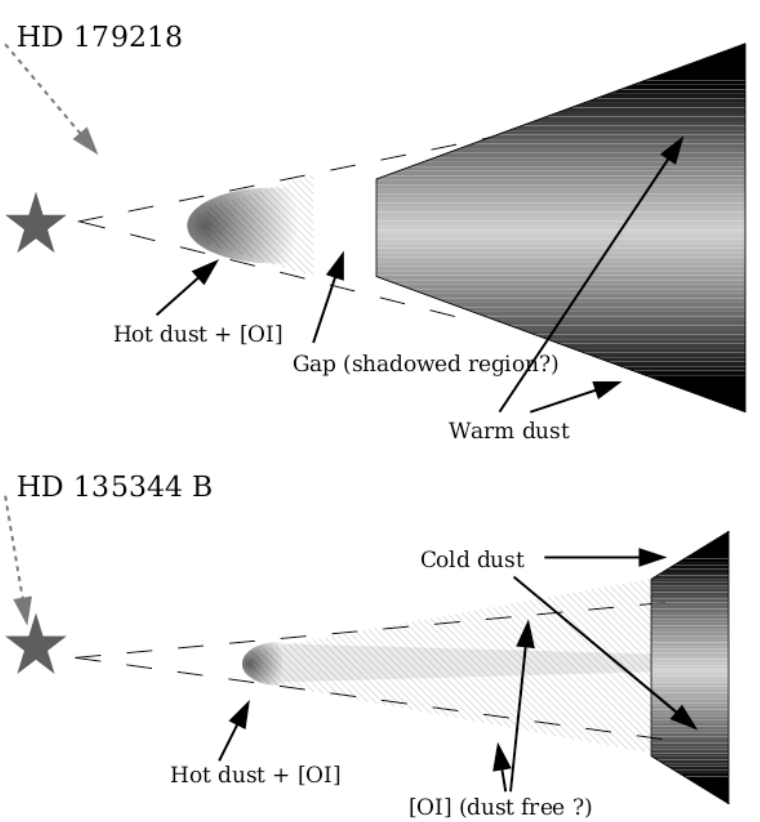

HD 101412

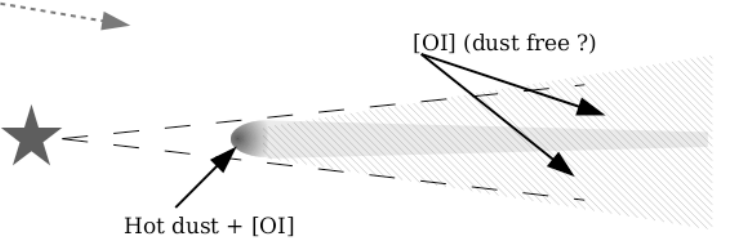

$\mathrm{R}$

Fig. 11. Simplified sketch of the disk around the three program stars. The regions where the dust and [O I] emissions are expected to arise are indicated. The inclination of the disk to the line of sight is shown by the dashed arrow at the upper-left corner. $H D$ 179218: the disk is large and flared. No dust emission is detected between 3-15 AU. Most of the silicate mid-infrared emission arises from the warm dust located in the surface layers of the outer part while the gas emission is mainly confined within $10 \mathrm{AU}$ to the star. $H D 135344 \mathrm{~B}$ : the $10 \mu \mathrm{m}$ emission arises from a compact region close to the star. Cold dust is detected at longer wavelengths. [O I] $6300 \AA$ emission is detected within the $10 \mu \mathrm{m}$ emitting region and further away at 5-10 AU where no dust emission is detected. $H D$ 101412: a compact dust ring that directly faces the star is responsible for the mid-infrared excess. The [O I] $6300 \AA$ line arises from the same region. Beyond this ring, the disk is shadowed. A dustfree flaring layer emerges from the shadow at larger radii. In all three cases, gas is likely present within the dust sublimation radius.

centered at $\sim 3-6 \mathrm{AU}$. The difference between gas and dust emitting regions for HD 179218 may reflects a real different structure for the two components or might be caused by other effects such as: 1) the action of chemical processes that reduce the abundance of $\mathrm{OH}$ in the outer part of the disk (in this case plenty of gas may still exist but we are not able to see it); 2) a contrast effect of the $[\mathrm{OI}]$ emission. In the latter case the $[\mathrm{OI}]$ emission is much stronger in the inner disk and it may outshine the [O I] from the outer disk. The presence of a dust gap needs further investigation as it may indicate possible ongoing planet formation.

HD 135344 B and HD101412 show a compact $10 \mu \mathrm{m}$ emitting region. The oxygen instead has a double peak brightness radial profile with the first peak coincident with the dust emission 
and a second peak further away from the star at $\sim 5-10 \mathrm{AU}$. In the case of HD $135344 \mathrm{~B}$ extended dust emission is found at $20.5 \mu \mathrm{m}$ (Doucet et al. 2006). Modelling the SED of HD 135344 B, Brown et al. (2007) propose a "cold-disk" geometry where the disk is mostly void of dust between $0.4-45$ AU. Dust is present within the inner $0.4 \mathrm{AU}$ and outside $45 \mathrm{AU}$. This is in agreement with our result in the sense that we detect a compact midinfrared dust emission; the dust at larger radii is cold and radiates at wavelengths longer than those traced by our observations. We suggest that the second [O I] peak arises either from a dust gap or from a dust-free layer above the shadow of the inner rim.

Following the phenomenological classification of Meeus et al. (2001), HD 101412 is a group II source, i.e. the disk is flat and self-shadowed in the dust. At the dust sublimation radius the disk is puffed-up and shadows the outer disk, which is not reached by the stellar UV radiation. The second peak of the [O I] emission (Fig. 8) is not expected for such a self-shadowed geometry. We suggest that this is the result of a flat disk geometry for the dust and a flared geometry for the gas. This might be the signature of a different scale height, and hence vertical structure, between gas and dust beyond the inner rim. This means that - in this object - gas and dust are physically decoupled in the surface layers of the disk allowing the gas to emerge from the shadow of the inner rim. If this is confirmed, it may have important consequences for the disk evolution: once gas and dust decouple the dust grains settle faster towards the mid-plane. The dust settling favors the physical separation between gas and dust until all the dust settles towards the mid-plane (Dullemond \& Dominik 2004) eventually leaving a region of gas in the upper layers of the disk. We thus suggest that HD 179218, HD 135344 B and HD 101412 form an evolutionary sequence where the disk, initially flared, becomes flat under the combined action of gas-dust decoupling, grain growth and dust settling. Interestingly, Acke et al. (2004) already found that cold grains in the mid-plane of the disk have grown to considerably larger sizes in Meeus et al., group II sources than in group I sources, suggesting that disks may evolve from a flared to a self-shadowed geometry. Similarly, Bouwman et al. (2008) find a correlation between the strength of the amorphous silicate feature and the shape of the SED, consistent with the settling of dust as a consequence of grain growth. What we add to this picture is a completely independent piece of evidence supporting this and perhaps the identification of the driving mechanism of this process.

Acknowledgements. D. Fedele acknowledges support by Deutscher Akad. Austauschdienst. (DAAD). G.M. acknowledges financial support by the Deutsche Forschungsgemeinschaft (DFG) under grant ME2061/3-1 and 3-2. We are grateful to the ESO staff in Garching and on Paranal for performing the MIDI observations in service mode. We thank the anonymous referee for many useful comments and suggestions which helped to improve this manuscript.

\section{Appendix A: Extended PAH emission}

Emission from polycyclic aromatic hydrocarbons (PAHs) is typically detected towards disks around Herbig stars (e.g. Meeus et al. 2001; Acke \& van den Ancker 2004; Sloan et al. 2005 \& Geers et al. 2007) and in some T Tauri stars (e.g. Geers et al. 2006, 2007). PAHs are heated by UV-photons from the central star. In many cases, the PAH emission is found to be spatially extended (e.g. van Boekel et al. 2004; Habart et al. 2004, 2006; Visser et al. 2007; Doucet et al. 2007 \& Boersma et al. 2008). In particular, in Herbig stars, the PAH emission is more extended than the continuum (at similar wavelengths) and can be as extended as the total size of the disk (Visser et al. 2007). In disks around less massive stars, given the lower UV flux, the emission is weaker and more compact around the central star. Three PAH emission features are present in the spectral range covered by our observations centered respectively at $8.6 \mu \mathrm{m}, 11.3 \mu \mathrm{m}$ and $12.7 \mu \mathrm{m}$. All the three sources we study here show evidence of emission of (at least one of) these features (e.g. van Boekel et al. 2005, and Fig. 1). The PAH emitting region may be much larger than the continuum/silicate one (Sect. 4.2). As a consequence, at the position of the PAH emission there are (at least) two visibility components which contribute to the MIDI observed visibility. Depending on the relative contribution of PAH emission to the total flux at a specific wavelength, the total visibility tends to be lower due to the presence of the extended PAH emission. Given the contamination of telluric absorption corresponding to the $12.7 \mu \mathrm{m}$ emission band, we do not take this feature into account in our analysis. The main findings that emerge from the analysis of the MIDI visibility and correlated flux are:

\section{PAH $8.6 \mu \mathrm{m}$ feature}

1. HD 101412: the visibility increases monotonically between $8 \mu \mathrm{m}$ and $9 \mu \mathrm{m}$ and the disk appears slightly more extended shortward of $9 \mu \mathrm{m}$ than at $10 \mu \mathrm{m}$. This may be a signature of extended PAH emission.

2. HD $135344 \mathrm{~B}$ : no evidence of $8.6 \mu \mathrm{m}$ emission in the total spectrum or in any of the correlated spectra.

3. HD 179218: the visibility is systematically low shortward of $9 \mu \mathrm{m}$. However, it is not clear whether this just reflects the characteristic sinusoidal modulation of the "ring-like" structure or whether it is the PAH emission that causes this effect.

PAH $11.3 \mu \mathrm{m}$ feature

1. HD 101412: a drop in the visibility of HD101412 around $11.3 \mu \mathrm{m}$ is detected along the second baseline $(59 \mathrm{~m}$, $102^{\circ}$ ). This is also the observation with the best signalto-noise ratio for HD 101412. A similar trend is visible along the third baseline $\left(62 \mathrm{~m}, 110^{\circ}\right)$, although the large error bars make this detection uncertain. HD 101412 is classified as a group II (self-shadowed) disk. The detection of PAH emission more extended than the dust emitting region is peculiar for such a disk structure. The UVexcited PAH molecules should lie in a dust-free surface layer of the disk. This source has a strong [O I] $6300 \AA$ emission - equally unusual for a group II source - which led van der Plas et al. (2008) to consider HD 101412 to be somewhat transitional in character between group I and group II.

2. HD 135344 B: a clear drop in visibility in the vicinity of $11.3 \mu \mathrm{m}$ is detected with MIDI with all baselines. This is even more clear in the correlated flux (Fig. 5): no emission is detected in the four measurements of the correlated flux, indicating that the $11.3 \mu \mathrm{m}$ PAH emission comes from spatial scales larger that those resolved by MIDI.

3. HD 179218: no detectable variation of the visibility is visible in the MIDI observations of HD 179218 around $11.3 \mu \mathrm{m}$. This disk is classified as a group I source (flared). The flaring geometry and the large size found for the dust emitting region may suggest that in this case dust and PAHs have a similar extent.

\section{Appendix B: Inclined ring model}

In the text, two simple geometrical models were used to fit the MIDI visibility: 1) a uniform disk; and 2) a uniform ring. The 
uniform disk model is a first-order approximation of a spherical brightness distribution. The intensity $(I(\rho, \phi), \rho$ and $\phi$ polar coordinates) is constant and non zero within a radius $\theta / 2$ and null outside:

$I(\rho, \phi)= \begin{cases}I_{0} & \rho \leq \theta / 2 \\ 0 & \rho>\theta / 2\end{cases}$

given the azimuthal symmetry, $I(\rho, \phi)=I(\rho)$. The Fourier transform of Eq. (B.1) is:

$V(u, v)=2 \frac{J_{1}(\pi \theta r)}{(\pi \theta r)}$

with $J_{1}$ Bessel function of the first order. The second model used in this paper is the uniform ring. In polar coordinates the brightness distribution of the uniform ring is:

$I(\rho)= \begin{cases}0 & \rho<\theta_{\text {in }} / 2 \\ I_{0} & \theta_{\text {in }} / 2 \leq \rho \leq \theta_{\text {out }} / 2 \\ 0 & \rho>\theta_{\text {out }} / 2\end{cases}$

with $\theta_{\text {in }}$ and $\theta_{\text {out }}$ inner and outer diameters of the ring.

Given the linearity property of the Fourier transform, the visibility of the uniform ring with inner size (diameter) $\theta_{\text {in }}$ and outer size $\theta_{\text {out }}$ is the difference of the visibility of two uniform disks. The visibility of a structure inclined by an angle $i$ and rotated by an angle $\phi$ (position angle of the major axis) can be obtained by rotating the $u, v$ coordinates (e.g. Berger \& Segransan 2007):

$u^{\prime}=u \cdot \cos (\phi)+v \cdot \sin \phi$

$v^{\prime}=-u \cdot \sin (\phi)+v \cdot \cos \phi$

and by applying a compression factor along the minor axis:

$r=\sqrt{u^{\prime 2}+v^{\prime 2} \cdot \cos (i)^{2}}$.

The visibility of an inclined uniform ring is:

$V_{\text {ring }}(r)=f_{\lambda}+2 \frac{1-f_{\lambda}}{\theta_{\text {out }}^{2}-\theta_{\text {in }}^{2}}\left[\theta_{\text {out }}^{2} \frac{J_{1}\left(\pi \theta_{\text {out }} r\right)}{\left(\pi \theta_{\text {out }} r\right)}-\theta_{\text {in }}^{2} \frac{J_{1}\left(\pi \theta_{\text {in }} r\right)}{\left(\pi \theta_{\text {in }} r\right)}\right]$

where $f_{\lambda}$ is the fractional contribution of an unresolved component (e.g. the central star) to the total flux at wavelength $\lambda$. In the extreme case of an infinitely thin ring with $\theta_{\text {in }}=\theta_{\text {out }}$, the visibility becomes:

$V_{\text {ring }}\left(\theta_{\text {in }}\right)=f_{\lambda}+\left(1-f_{\lambda}\right) J_{0}\left(\pi \theta_{\text {in }} r\right)$.

The model adopted for HD 179218 is the sum of two uniform rings:

$I(\rho)= \begin{cases}0 & \rho<\theta_{1} / 2 \\ I_{0} & \theta_{1} / 2 \leq \rho \leq \theta_{2} / 2 \\ 0 & \theta_{2} / 2<\rho<\theta_{3} / 2 \\ I_{0} & \theta_{3} / 2 \leq \rho \leq \theta_{4} / 2 \\ 0 & \rho>\theta_{4} / 2\end{cases}$ with $\theta_{1}, \theta_{2}, \theta_{3}$ and $\theta_{4}$ the diameters of the two uniform rings respectively. The visibility of such a model is:

$V(r)=f_{\lambda} V_{U R, \text { in }}\left(\theta_{1}, \theta_{2}\right)+\left(1-f_{\lambda}\right) V_{U R, \text { out }}\left(\theta_{3}, \theta_{4}\right)$

with $V_{U R_{\text {in }}}$ and $V_{U R_{\text {out }}}$ the visibility of a uniform ring (Eq. (B.7)) for the inner and outer component respectively.

\section{References}

Ábrahám, P., Mosoni, L., Henning, T., et al. 2006, A\&A, 449, L13

Acke, B., \& van den Ancker, M. E. 2004, A\&A, 426, 151

Acke, B., van den Ancker, M. E., \& Dullemond, C. P. 2005, A\&A, 436, 209

Berger, J. P., \& Segransan, D. 2007, New Astron. Rev., 51, 576

Boersma, C., Bouwman, J., Lahuis, F., et al. 2008, [arXiv: 0804 . 1199]

Bouwman, J., et al. 2008, [arXiv:0802 . 3033]

Brown, J. M., Blake, G. A., Dullemond, C. P., et al. 2007, ApJ, 664, L107

Brown, J. M., Blake, G. A., Qi, C., Dullemond, C. P., \& Wilner, D. J. 2008, ApJ, 675, L109

Chesneau, O., Collioud, A., De Marco, O., et al. 2006, A\&A, 455, 1009

Cohen, M., Walker, R. G., Carter, B., et al. 1999 AJ, 117, 1864

D’Alessio, P., Calvet, N., \& Hartmann, L. 2001, ApJ, 553, 321

Dent, W. R. F., Greaves, J. S., \& Coulson, I. M. 2005, MNRAS, 359, 663

Doucet, C., Pantin, E., Lagage, P. O., \& Dullemond, C. P. 2006, A\&A, 460, 117

Doucet, C., Habart, E., Pantin, E., et al. 2007, A\&A, 470, 625

Dullemond, C. P., \& Dominik, C. 2004, A\&A, 421, 1075

Dullemond, C. P., Dominik, C., \& Natta, A. 2001, ApJ, 560, 957

Eisner, J. A., Lane, B. F., Hillenbrand, L. A., et al. 2004, ApJ, 613, 1049

Eisner, J. A., Hillenbrand, L. A., White, R. J., et al. 2007, ApJ, 669, 1072

Geers, V. C., Augereau, J.-C., Pontoppidan, K. M., et al. 2006, A\&A, 459, 545

Geers, V. C., van Dishoeck, E. F., Visser, R., et al. 2007, A\&A, 476, 279

Glassgold, A. E., Najita, J. R., \& Igea, J. 2007, ApJ, 656, 515

Gorti, U., \& Hollenbach, D. 2007, in preparation

Habart, E., Natta, A., \& Krügel, E. 2004, A\&A, 427, 179

Habart, E., Natta, A., Testi, L., \& Carbillet, M. 2006, A\&A, 449, 1067

Henning, Th. 2007, Early Phases of Planet Formation in Protoplanetary Disks: Nobel Symp., in press

Isella, A., \& Natta, A. 2005, A\&A, 438, 899

Jaffe, W. J. 2004, Proc. SPIE, 5491, 715

Johansen, A., Dullemond, Brauer, F., C., Klahr, H., \& Henning, T. 2008, A\&A, 486, 597

Jonkheid, B., Faas, F. G. A., van Zadelhoff, G.-J., \& van Dishoeck, E. F. 2004, A\&A, 428, 511

Kamp, I., \& Dullemond, C. P. 2004, ApJ, 615, 991

Lahuis, F., van Dishoeck, E. F., Blake, G. A., et al. 2007, ApJ, 665, 492

Leinert, Ch., Graser, U., Przygodda, F., et al. 2003, Ap\&SS, 285, 73

Leinert, C., van Boekel, R., Waters, L. B. F. M., et al. 2004, A\&A, 423, 537

Liu, W. M., Hinz, P. M., Meyer, M. R., et al. 2007, ApJ, 658, 1164

Meeus, G., Waters, L. B. F. M., Bouwman, J., et al. 2001, A\&A, 365, 476

Millan-Gabet, R., Schloerb, F. P., \& Traub, W. A. 2001, ApJ, 546, 358

Monnier, J. D., Berger, J.-P., Millan-Gabet, R., et al. 2006, ApJ, 647, 444

Natta, A., Testi, L., Calvet, N., et al. 2007, Protostars and Planets V, 767

Nomura, H., \& Millar, T. J. 2005, A\&A, 438, 923

Pascucci, I., Hollenbach, D., Najita, J., et al. 2007, ApJ, 663, 383

Quanz, S. P., Henning, T., Bouwman, et al. 2006, ApJ, 648, 472

Ratzka, T. Ph.D. Thesis, Ruprecht-Karls-Universität Heidelberg

Sloan, G. C., Keller, L. D., Forrest, W. J., et al. 2005, ApJ, 632, 956

van Boekel, R., Waters, L. B. F. M., Dominik, C., et al. 2004, A\&A, 418, 177

van Boekel, R., Min, M., Waters, L. B. F. M., et al. 2005, A\&A, 437, 189

van der Plas, G., van den Ancker, M. E., Fedele, D., et al. 2008, A\&A, 485, 487

(Paper I)

Visser, R., Geers, V. C., Dullemond, C. P., et al. 2007, A\&A, 466, 229 